UCLA-APH0069-7/93

\title{
Prospects for Detection of a Cosmologically Significant Neutrino Mass from a Galactic Supernova Neutrino Burst Using a Neutral-Current-Based Detector
}

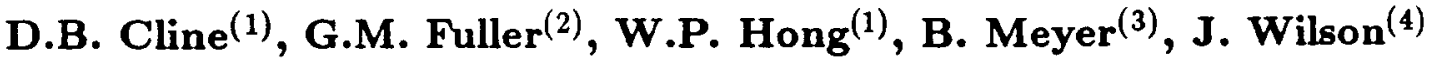 \\ (1) Department of Physics \& Astronomy, University of California, Los Angeles, CA 90024 \\ (2) Department of Physics, University of California at San Diego, La Jolla, CA 92099 \\ (9) Department of Physics \& Astronomy, Clemson University, Clemson, SC, 29694 \\ (4) Lawrence Livermore National Laboratory, Box 808 L-419, Livermore, CA 94550
}

\begin{abstract}
We discuss how proposed supernova neutrino detectors could measure masses for $\nu_{\mu}$ or $\nu_{\tau}$ neutrinos in the range of $15 \mathrm{eV}$ to $50 \mathrm{eV}$. The range for measurable masses might be extended down to $5 \mathrm{eV}$, depending on our confidence in some of the predicted features of the supernovaneutrino-burst signal. We discuss the expected characteristics of supernova neutrino signals in proposed neutral-current-based detectors.
\end{abstract}

PACS numbers: $98.80 . \mathrm{Cq}, 98.80 . \mathrm{Dr}, 98.60 . \mathrm{A}, 12.15 .-\mathrm{Ji}, 13.15 .-\mathrm{f}$

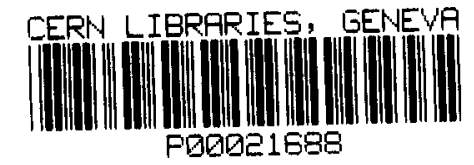




\section{Introduction}

The issue of whether or not neutrinos have masses is important for astrophysics and cosmology. Unfortunately, terrestrial experimental probes of neutrino mass, especially the $\nu_{\mu}$ and $\nu_{\tau}$ masses, remain problematic. Astrophysical considerations may represent the best hope for inferring neutrino masses and mixings. In this paper we examine how proposed neutralcurrent-based supernova neutrino burst detectors, in conjunction with the next generation water Čerenkov detectors, could use a galactic supernova event to either measure or place constraints on $\nu_{\mu}$ and/or $\nu_{\tau}$ masses in excess of $5 \mathrm{eV}$. Such measurements would have important implications for our understanding of particle physics, cosmology, and the solar neutrino problem, and would be complimentary to proposed laboratory vacuum oscillation experiments.

A light neutrino mass between $1 \mathrm{eV}$ and $100 \mathrm{eV}$ would be highly significant for cosmology [1] . In fact if a neutrino contributes a fraction $\Omega_{\nu}$ of the closure density of the universe it must have mass $m_{\nu} \approx 92 \Omega_{\nu} h^{2} \mathrm{eV}$, where $h$ is the hubble parameters in units of $100 \mathrm{~km}^{-1} \mathrm{sec}^{-1} \mathrm{Mpc}^{-1}$. Reasonable ranges for $\Omega_{\nu}$ and $h$ then give $1 \mathrm{eV}$ to $92 \mathrm{eV}$ as a cosmologically significant range. A neutrino with a mass in the higher end of this range, i.e. $10 \leqslant m_{\nu} \leqslant 92 \mathrm{eV}$, could contribute significantly to the closure density of the universe. The Cosmic Background Explorer (COBE) observation of anisotropy in the microwave background [2], combined with observations at smaller scales [3] , and the distribution of galaxy streaming velocities, have been interpretated as implying that there are two components of dark matter, i.e. hot and cold dark matter components, $\Omega_{\mathrm{CDM}} \sim 0.6$ and $\Omega_{\mathrm{HDM}} \sim 0.3$. The hot dark matter $(\mathrm{HDM})$ component could be provided by a neutrino with a mass of about or greater than $7 \mathrm{eV}$ [4] .

Calculations of the standard solar model $\nu_{e}$ flux [5] are not easily reconciled with the results of recent experiments [6] . This constitutes the solar neutrino problem. Matter enhanced neutrino flavor transformation remains an attractive mechanism to solve this problem. This mechanism is referred to as the Mikheyev-Smirnov-Wolfenstein (MSW) solution [7]. The favored MSW solution imply that the $\nu_{e} \nu_{\mu}$ (or $\nu_{e} \nu_{\tau}$ ) mixing angle is confined to two small regions: the so-called large angle region with $\sin ^{2} 2 \theta \approx 0.1$; and another region with $\sin ^{2} 2 \theta \approx$ 
$10^{-2}$. In either case the difference in the squares of the neutrino mass eigenvalues must be about $\delta m^{2} \approx m_{1}^{2}-m_{2}^{2} \simeq 10^{-5}-10^{-6} \mathrm{eV}^{2}$. This suggests that the mass of the $\nu_{\mu}$ or $\nu_{\tau}$ neutrinos needs to be of order $m_{\nu_{\mu(r)}} \approx 2-3 \times 10^{-3}$. The "seesaw" mechanism [8] for generating a muon neutrino masses in this range also suggests $\nu_{\tau}$ masses in the cosmologically significant range.

Despite the cosmological and particle physics interest in massive neutrinos there are very few terrestrial experimental means for measuring neutrino masses. The electron neutrino mass is constrained by the tritium endpoint experiments [9] to be less than about $7.2 \mathrm{eV}$. It is conceivable that $\nu_{\mu} \nu_{\tau}$ accelerator neutrino oscillation experiments, such as the NOMAD [10] and CHORUS [11] experiments at CERN, could be used to infer a mass in the cosmologically interesting range for $\nu_{\mu}$ or $\nu_{\tau}$. This would depend on there being a fairly large vacuum mixing between these neutrino flavors. Nucleosynthesis from supernovae could possibly provide a signature for neutrinos with these masses [12] .

Perhaps the most straightforward and obvious signature of a massive neutrino would come from the lengthening in flight time from a distant supernova. For example, the flight time difference between $\nu_{\tau}$ and $\nu_{e}\left(\bar{\nu}_{e}\right)$ in seconds is

$$
\delta t \approx 0.514 R_{10 \mathrm{kpc}}\left[\left(\frac{m_{\nu_{\tau}}}{E_{\nu_{\tau}}}\right)^{2}-\left(\frac{m_{\bar{\nu}_{e}}}{E_{\bar{\nu}_{e}}}\right)^{2}\right],
$$

where $m_{\nu_{\tau}}$ is in $\mathrm{eV}, E_{\nu_{\tau}}$ is the neutrino energy in $\mathrm{MeV}$, and $R_{10 \mathrm{kpc}}$ is the distance to the supernova in units of $10 \mathrm{kpc}$. A finite neutrino mass would alter the neutrino spectra in characteristic ways which could result in broadening and flattening of the observed signal $[15,16]$ Thus neutrino masses might be obtained by comparing the observed neutrino signal with the signal expected from supernova models. However the paucity of observed neutrino events (19 events) detected by the Kamiokande II (KII) [13] and the Irvine-Michigan-Brookhaven (IMB) [14] collaboration for the SN 1987A explosion does not allow us to obtain a clear signature for a massive $\bar{\nu}_{e}$. Since detectors such as Super-Kamiokande (SK) and IMB are relatively insensitive to $\nu_{\mu}$ and $\nu_{\tau}$, they are unlikely to measure cosmologically significant neutrino masses for these flavors. One of the neutral current based detectors being built at 
present, the Sudbury Neutrino Observatory (SNO) [17], seems to have some capabilities to detect $\nu_{\tau}$ events (see Table II and Table IV of Ref. [16] ) but it would not be likely to give clean massive neutrino signatures [18] .

The proposed Supernova Neutrino Burst Observatory (SNBO [21] ), working in conjunction with SK, could give clear signatures for $\nu_{\mu}$ and/or $\nu_{\tau}$ when the masses of these species are in the cosmologically significant range. As we will discuss however, this will be dependent on having small mixings with $\nu_{e}$.

This conclusion is in contrast to previous pessimistic conclusions [19] regarding detection of such neutrino masses. The pessimistic conclusions of the study in ref [19] are largely based on the limitation in size of a massive detector and intrinsic backgrounds. In section II, we review two methods for measurement of neutrino masses utilizing charged and neutral current detectors. We also discuss all existing and proposed detectors' characteristics and capabilities. In section III, we calculate inelastic neutrino-nucleus interaction cross sections and estimate the expected supernova neutrino burst signal in large SNBO-like neutral current-based detectors. We consider the analysis of expected supernova neutrino burst signals in section IV. 
II. Review of neutrino time of flight mass determination from SN: Two techniques

In principle there are two basic methods for neutrino mass determination using finite time delay from a distant supernova. The first method is to analyze the total observed neutrino signal using parameterized supernova models. The neutrino mass is estimated from the model signal which gives the greatest likehood for agreement with the observed signal. The supernova 1987A data from the Kamiokande and the IMB detectors, which use the charged current reaction $\left(\bar{\nu}_{e}+p\right)$, at best put an upper bound on the $\nu_{e}$ neutrino mass $(<9.3 \mathrm{eV}(95 \%$ C.L) [20] ). Note that the signal dispersion due to time-of-flight effects in a water Čerenkov detector is given as:

$(\delta t)_{\text {dispersion }}=0.0514 \times \mathrm{R}_{10 \mathrm{kpc}} \frac{\int_{0}^{\infty}\left(\frac{m_{\nu_{e}}}{\epsilon}\right)^{2} \epsilon^{2} D_{e}(\epsilon) \exp \left(-\epsilon / T_{\nu_{e}}\right) d \epsilon}{\int_{0}^{\infty} D(\epsilon) \epsilon^{2} \exp \left(-\epsilon / T_{\nu_{e}}\right) d \epsilon} \simeq(0.03 \mathrm{sec}) \mathrm{R}_{10 \mathrm{kpc}}\left(m_{\nu_{e}} / T_{\nu_{e}}\right)^{2}$,

where $D_{e}(\epsilon)$ is the detector efficiency multiplied by the cross section for a water Čerenkov reaction, $m_{\nu_{e}}$ is the $\nu_{e}$ neutrino mass in $\mathrm{eV}$, and $T_{\nu_{e}}$ is the temperature of the black body neutrino spectrum in $\mathrm{MeV}$. The major difficulty in deconvolving a clear massive $\nu_{e}\left(\bar{\nu}_{e}\right)$ signal from the measured spectrum is that the electron neutrino has a time-of-flight dispersion for a galactic supernova of $(\delta t)_{\text {dispersion }} \simeq 0.3 \mathrm{sec}$ for $R_{10 \mathrm{kpc}}=10, m_{\nu_{e}}=10 \mathrm{eV}$, and $T_{\nu_{e}}=3 \mathrm{MeV}$. This mass-induced dispersion is comparable to the intrinsic signal dispersion.

Fortunately, when the Super-Kamiokande detector, LVD, and SNO are on line for a supernova watch in the future, high statistics measurements during the first second of the signal may show the characteristic increase in rise time for massive $\nu_{e}\left(\bar{\nu}_{e}\right)$. For example, a 50 msec rise to maximum in KII and LVD at zero mass becomes a $300 \mathrm{msec}$ rise time for a neutrino with a mass of $10 \mathrm{eV}[16]$. Thus high statistics measurements are the key for measuring $\nu_{e}\left(\bar{\nu}_{e}\right)$ masses in the cosmologically significant range. This method of neutrino mass measurement can not be extended to $\nu_{\mu}$ or $\nu_{\tau}$ unless a neutral-current-based detector is employed.

The second method of neutrino mass determination using a supernova neutrino burst event is suitable for $\nu_{\mu}$ and/or $\nu_{\tau}$ neutrino mass measurements. In this method, information from 
other water detectors sensitive to $\bar{\nu}_{e}$ (for example Super-Kamiokande) is used to give the time of a stellar collapse. The time shift in the onset of the $\nu_{\mu}$ or $\nu_{\tau}$ signal in a pure neutral-currentbased detector such as SNBO [21] is then used to infer or constrain the $\nu_{\mu}$ or $\nu_{\tau}$ mass. The $\nu_{\mu}$ and $\nu_{\tau}$ luminosities rise very rapidly after stellar core bounce. Figure 1 shows neutrino luminosities as a function of time for a typical supernova event. This figure shows luminosity histories for $\bar{\nu}_{e}, \nu_{e}$, and $\nu_{\mu}\left(\nu_{\tau}\right)$. Signal time shifts of the order of $\sim 0.1 \mathrm{sec}$ are detectable, so that collapse events in our galaxy $\left(\mathrm{R}_{\mathrm{kpc}} \approx 10-20\right)$ are far enough away to give measurable shifts for $\nu_{\mu}$ or $\nu_{\tau}$ with cosmologically significant masses.

It is plausible that the detection of gravitational waves could be used as the initial time mark for a pure neutral current detector, since the gravity wave signal would be generated within $\sim 0.001 \mathrm{sec}$ of the stellar core bounce. The energy output in gravity waves in stellar collapse is estimated to be rather low, but very high sensitivity gravitational wave [22] detectors should be operating for supernova watch in the future.

Several reactions can be employed for supernova neutrino burst detection. Table 1 (also see Burrows et al. [16] ) summarizes these reactions, and possible detection schemes employing them, for several detectors in the operation or construction stage. There are three possible reactions which can be used to detect $\nu_{x}\left(\bar{\nu}_{x}\right)$ neutrinos $(x=e, \mu, \nu)$ :

$$
\left\{\begin{array}{l}
\stackrel{(-)}{\nu}_{x}+e \rightarrow \stackrel{(-)}{\nu}_{x}+\mathrm{e}: \text { elastic scattering on electrons } \\
\stackrel{(-)}{\nu}_{x}+N \rightarrow \stackrel{(-)}{\nu}_{x}+\mathrm{N}: \text { elastic scattering on Nucleons or Nuclei } \\
\stackrel{(-)}{\nu}_{x}+N \rightarrow \stackrel{(-)}{\nu}_{x}+\mathrm{N}^{*}: \text { inelastic scattering on Nuclei }
\end{array}\right.
$$

where $\mathrm{N}^{*}$ is an excited nuclear state that results in the production of a proton, a neutron, or a hard photon that is subsequently detected. In all cases the major sources of possible confusion with $\nu_{e}\left(\bar{\nu}_{e}\right)$ induced events come from the reactions,

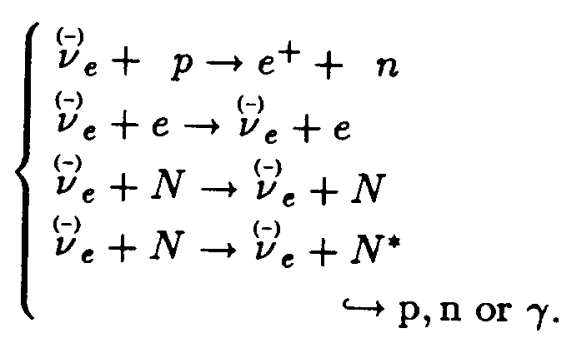


Thus the only unique way to separate the $\stackrel{(-)}{\nu}_{\mu, \tau}$ and $\stackrel{(-)}{\nu}_{e}$ processes is through the flight time difference between these species.

Table 2 lists the approximate event rates for various channels for supernova detection in the 1990's and beyond (for a detailed review see Burrows et al. [16] ). The primary conditions for successful supernova neutrino burst detection are that the detector be large in effective volume and be composed of inexpensive material with high neutrino interaction cross-section. Most detectors in operation now or proposed for the future rely on large $\left(\bar{\nu}_{e}\right)$ cross-section, $\bar{\nu}_{e}+p \rightarrow n+e^{+}$(KII,SK,IMB,LVD, MACRO). The ICARUS [24] detector is in the construction stage, and will provide some important information regarding the $\nu_{e}$ burst from supernovae resulting from the shock breakout phase of neutrino emission in supernova core. Unfortunately, the ICARUS detector is not optimized for detecting $\nu_{\mu}$ and $\nu_{\tau}$. At present, the most promising neutral current detector actually being constructed is SNO [17], which utilizes the neutral-current breakup reaction of deuterium: $\nu_{x}+d \rightarrow n+p+\nu_{x}$, where $x=e, \mu, \tau$. However the charged current reactions $\nu_{e}+d \rightarrow p+p+e^{-}$and $\bar{\nu}_{e}+d \rightarrow n+n+e^{+}$ may contaminate the massive $\nu_{\mu}$ or $\nu_{\tau}$ signals (Burrowet al. [16] discusses this point). The most useful neutral current based galactic supernova detector may be SNBO. This detector sited in a low radioactive background would give a large number of $\nu_{\tau}\left(\nu_{\mu}\right)$ events - about 10,000 depending on detector geometry and the detector medium (see Sec III). 


\section{Neutrino mass determination}

\section{A. Neutrino Detection Employing Neutrino-Nucleus Inelastic Scattering}

A promising technique for neutrino detection involves neutral current inelastic neutrinonucleus scattering. If this reaction is endothermic (neutrino scatters to a lower energy state) then the nucleus may be left in a particle unstable exited state:

$$
\nu_{x}+A(Z, N) \rightarrow A(Z, N-1)+n+\nu_{x}^{\prime}
$$

where the nucleus has mass $A=Z+N$, and contains $Z$ protons and $N$ neutrons. In the process shown in equation (5) the exited nucleus decays by neutron emission. In this expression $\nu_{x}$ is either a $\nu_{e}, \nu_{\mu}, \nu_{\tau}$, or their antiparticles. Recent studies have shown that the cross section for this process can be very large by nuclear weak interaction standards, approaching $\sigma_{n} \approx$ $10^{-42} \mathrm{~cm}^{2}$ per nucleon for the energetic $\nu_{\mu}$ and $\nu_{\tau}$ neutrinos expected from stellar collapse [25,26]

In fact the cross section for the interaction in equation (5) is quite energy dependent, so that the $\nu_{\mu}$ and $\nu_{\tau}$ neutrinos dominate the neutron yield. In effect then, a detector based on this inelastic process filters out low energy neutrinos. We expect that the average energies for the neutrino species satisfy $\bar{\epsilon}_{\nu_{\mu(\tau)}}>\bar{\epsilon}_{\bar{\nu}_{e}}>\bar{\epsilon}_{\nu_{e}}$. Typical values for these quantitative after 1 seconds are $\bar{\epsilon}_{\nu_{\mu(\tau)}} \approx 25 \mathrm{MeV}, \bar{\epsilon}_{\bar{\nu}_{e}} \approx 16 \mathrm{MeV}, \bar{\epsilon}_{\nu_{e}} \approx 11 \mathrm{MeV}$. In the absence of significant

neutrino flavor mixing between $\stackrel{(-)}{\nu}_{\tau(\mu)}$ and $\stackrel{(-)}{\nu}_{e}$, we can conclude that detectors utilizing reaction (5) operate as flavor filters, effectively detecting only $\stackrel{(-)}{\nu}_{\mu}$ and $\stackrel{(-)}{\nu}_{\tau}$ induced events.

We follow reference [26] and compute the total average neutrino-nucleus inelastic scattering cross section as

$$
\sigma_{\mathrm{FD}}\left(T_{\nu}\right)=\frac{\sigma_{0} \int_{0}^{\infty} F_{\nu}\left(E_{\nu}\right) d E_{\nu} \int_{0}^{E_{\nu}} d E_{\nu}^{\prime} E_{\nu}^{\prime 2} \beta\left(<E>, E_{\nu}-E_{\nu}^{\prime}\right)}{\int_{0}^{\infty} F_{\nu}\left(E_{\nu}\right) d E_{\nu}}
$$

where $\sigma_{0} \approx 2.583 \times 10^{-44} \mathrm{~cm}^{2} \mathrm{MeV}^{-2}$, and $E_{\nu}$ and $E_{\nu}^{\prime}$ are the incident and scattered neutrino energies, respectively. In this equation we present a neutrino-flux-averaged cross section, where $F_{\nu}\left(E_{\nu}\right)$ is the neutrino flux at energy $E_{\nu}$. The weak nuclear strength function 
is $\beta\left(\langle E\rangle, E_{\nu}-E_{\nu}^{\prime}\right)$, and $\left(E_{\nu}-E_{\nu}^{\prime}\right)$ is the neutrino energy transfer to the nucleus. Shell model fits for this strength function can be found in reference [26] .

Figure 2 shows total flux-averaged neutrino-nucleus inelastic scattering cross section per nucleon for ${ }^{28} \mathrm{Si}$ and ${ }^{23} \mathrm{Na}$ as function of $T_{\nu}$. In this figure we have assumed that each nucleus is in an exited state with an excitation energy of $\langle E>\approx 20 \mathrm{MeV}$. In fact, for inelastic neutrinonucleus scattering in the endothermic channel there is very little dependence of the cross section on nuclear excitation energy, so that the results shown in Figure 1 would be essentially unchanged for $\left\langle E>=0\right.$. The neutrino flux $F_{\nu}\left(E_{\nu}\right)$ used for the results in Figure 2 is taken to be a black body Fermi-Dirac distribution with zero chemical potential and temperature $T_{\nu}=5$ $\mathrm{MeV}$.

We can use these techniques to make estimates of the neutron production cross sections per nucleon for various materials which might serve as a detection medium for a neutral current based supernova neutrino burst detector. We have computed these cross sections for three different "rocks" composed of $\mathrm{NaCl}, \mathrm{CaCO}_{3}$, and $\mathrm{SiO}_{2}$. We sum the cross section for each nucleus, weighting each by the appropriate frequency of occurrence in each molecule. Obviously, a terrestrial detector has nuclear excitation energy $\langle E\rangle=0$, and the appropriate weak strength function is $\beta\left(0, E_{\nu}-E_{\nu}^{\prime}\right)$. The neutron production cross section is then,

$$
\sigma_{\mathrm{n}}^{i}\left(E_{\nu}\right)=\alpha_{i} \sigma_{0} \int_{0}^{E_{\nu}} d E_{\nu}^{\prime} E_{\nu}^{\prime 2} \beta_{i}\left(0, E_{\nu}-E_{\nu}^{\prime}\right),
$$

where the index $i$ refer to a specific nucleus, and $\alpha_{i}$ is the efficiency for neutron emission.

In Figure 3 we show the neutron emission cross section per nucleon as a function of incident neutrino average energy for detector material composed of $\mathrm{NaCl}, \mathrm{CaCO}_{3}$, and $\mathrm{SiO}_{2}$ (dashed, dotted, and dot-dashed curves, respectively). In these calculations we consider a neutron emission efficiency of $\alpha \approx 20 \%$ for all nuclear species. Shown for comparison purposes is the cross section for $\bar{\nu}_{e}$ absorption on protons, $\bar{\nu}_{e}+p \rightarrow n+e^{+}$. It is apparent from this figure that the neutron production cross sections will dominate the $\bar{\nu}_{e}+p \rightarrow n+e^{+}$cross section when the incident neutrino energy is in excess of $25 \mathrm{MeV}$. Given the average neutrino energy 
hierarchy discussed above it is clear that $\stackrel{(-)}{\nu}_{\mu}$ and $\stackrel{(-)}{\nu}_{\tau}$ neutrinos will dominate neutron production in neutral current based detectors. This conclusion is true, however, only when $\stackrel{(-)}{\nu}_{\mu(\tau)} \rightarrow \stackrel{(-)}{\nu}_{e}$ interconversion in negligible.

\section{B. Expected Performance for a Neutral Current Based Neutrino Detectors}

In gauging the prospects for time of flight delay measurement for $\nu_{\mu}$ or $\nu_{\tau}$ mass from a galactic supernova event it is useful to consider the characteristics of a plausible neutral current based detector. As described above such a detector is sensitive primarily to high energy neutrinos and, therefore, acts as a neutrino flavor filter in the absence of matter enhanced oscillations. The proposed Supernova Neutrino Burst Observatory, or SNBO (see references [21] and [23] ) would use $\mathrm{NaCl}$ in salt deposit as a detector medium. The high threshold energy $\left(E_{\mathrm{th}} \gtrsim 12 \mathrm{MeV}\right.$ ) for neutron production in ${ }^{23} \mathrm{Na}$ enhances the flavor aspects of the detector. This high threshold is a result of the neutron separation energies for ${ }^{23} \mathrm{Na}$ and ${ }^{35} \mathrm{Cl}$. The SNBO detector would use $\mathrm{BF}_{3}$ neutron counters, which detect neutrons from neutrino-nucleus spallation events via the reaction,

$$
n+{ }^{10} \mathrm{~B} \rightarrow{ }^{7} \mathrm{Li}+{ }^{4} \mathrm{He} .
$$

Clearly, the feasibility of such a detector depends on having low neutron backgrounds. At least one site has been suggested where the background is measured to be low enough that the detector would provide a large number of counts from a galactic supernova. This is the WIPP site (Waste Isolation Pilot Plant [23] ) where the neutron background has been measured to be about six counts per hour for a two meters long neutron counter(see reference [28] )

A detector like SNBO is envisaged to consist of a tunnel serval thousand feet under ground (to reduce cosmic ray background) and of under $1 \mathrm{~km}$ long and 5 meters in diameter. This tunnel would be lined with $\mathrm{BF}_{3}$ counters. The detailed Monte-Carlo calculation of neutron transportation and efficiency for neutron detection indicate that of order $10^{4}$ high-energyneutrino-induced events would be expected from a supernova at $10 \mathrm{kpc}$ distance (i.e., a galactic supernova). 
Low neutron background is essential for massive neutrino time of flight delay measurement for another reason. These measurements would require time correlation between neutron detection and the neutrino burst. Monte Carlo Calculations show that $90 \%$ of the neutrons counted come in the first millisecond after emission from the excited nuclei. The neutrino signals are expected to have duration of about 10 seconds, with peak luminosities occurring within the first few hundreds of milliseconds.

\section{Expected Supernova Signals and Analysis}

We briefly discuss some essential features of neutrino emission from supernova and how these features relate to time of flight delay measurement. The numerical works of Wilson [30] , Wilson and Mayle [31], Bruenn [32] , Myra and Burrows [33], and Burrows [34] show that there are two short pulses of neutrinos followed by a much longer pulse from a Type II supernova. As shown in Figure 1, electron neutrinos $\left(\nu_{e}\right)$, emitted predominantly during the infall and when the shock reaches the neutrino-sphere, carry away an energy of a few times $10^{51}$ ergs. The "neutronization-pulse" comes when the shock passes through the neutrinosphere. Subsequently, the long thermal neutrino pulse is characterized by all six neutrino species carrying almost all the gravitational binding energy $\left(\sim 10^{53}\right.$ ergs $)$ of the new born neutron star. The cooling and deleptonization of the photo-neutron star occur largely during this phase.

The flight time shift method is somewhat dependent on the particular collapse model. The computer model developed by Wilson and Mayle [31] has given good agreement with the observation of SN 1987A. The calculations give the correct neutrino spectrum and time distribution as well as the observed total explosion energy. A key property which we require from these calculations is the rise time of the $\bar{\nu}_{e}$ emission relative to the $\nu_{\mu, \tau}$ emission. The $\bar{\nu}_{e}$ production and emission is suppressed until the core becomes hot enough that degeneracy is decreased. Similarly, the $\nu_{\mu, \tau}$ emission is initially limited by the temperature since the $\nu_{\mu, \tau}$ production rate is proportional to $\mathrm{T}^{9}$. A temperature of about $9 \mathrm{MeV}$ is needed to produce enough $\nu_{\mu, \tau}$ so that the luminosity, $L_{\mu, \tau}$ is diffusion limited. This temperature is also about 
the same as that needed to relieve the degeneracy suppression of the $\bar{\nu}_{e}$. Thus, the coincidence of the rise time of the $\bar{\nu}_{e}$ and $\nu_{\mu, \tau}$ is not strongly model dependent. Note that the $\bar{\nu}_{e}$ and $\nu_{\mu, \tau}$ signals rise abruptly to their peak in about $0.1 \mathrm{sec}$ (see Figure 1). The model calculations of Myra and Burrows [33] also shows the narrow peak in the $\bar{\nu}_{e}$ and $\nu_{\mu, \tau}$ spectrum in the first 0.1-0.2 s after core bounce $[16,33]$.

Following the above outline we can estimate the expected count rate from a supernova burst for various detectors materials (or "rocks"). Considering the signal delay from finite neutrino masses, the count rates is estimated as,

$$
C(t)_{\text {rock }}=\frac{N_{T}}{4 \pi R_{10 \mathrm{Kpc}}^{2}} \frac{\int_{\epsilon_{\text {cut }}}^{\infty} \sigma_{\mathrm{n}}\left(\epsilon_{\nu_{\tau}}, \epsilon_{\mathrm{cut}}\right) \epsilon_{\nu_{\tau}}^{2} \exp \left(-\epsilon_{\nu_{\tau}} / T_{\nu_{\tau}}\left(t_{e}\right)\right) N_{\nu_{\tau}}\left(t_{e}\right) d \epsilon_{\nu_{\tau}}}{\int_{\epsilon_{\text {cut }}}^{\infty} \epsilon_{\nu_{\tau}}^{2} \exp \left(-\epsilon_{\nu_{\tau}} / T_{\nu_{\tau}}\left(t_{e}\right)\right) d \epsilon_{\nu_{\tau}}}
$$

where

$$
t_{e}=t-0.514 R_{10 \mathrm{kpc}}\left(\frac{m_{\nu_{\tau}}}{\epsilon_{\nu_{\mathrm{T}}}}\right)^{2} \mathrm{sec},
$$

where $N_{T}$ is the total number of target nucleons in various rocks, $\sigma_{n}\left(\epsilon_{\nu_{\tau}}, \epsilon_{\mathrm{c}}\right)$ is the neutron production cross sections per nucleon (see Figure 3), and where the cut-off energy is $\epsilon_{\mathrm{cut}} \simeq 20$ $\mathrm{MeV}$. Figure 4 shows average neutrino energies for $\bar{\nu}_{e}, \nu_{e}$ and $\nu_{\mu}\left(\nu_{\tau}\right)$ as function of time. The core temperature is taken as $T_{\nu_{\tau}}\left(t_{e}\right)=\bar{\epsilon}_{\nu_{\tau}} / 3$ and the total number of incident neutrinos $N_{\nu_{\tau}}\left(t_{e}\right)=L_{\nu_{\tau}} / \bar{\epsilon}_{\nu_{\tau}}$ is fitted to the computer model (see Figures 3 and 4), where $\bar{\epsilon}_{\nu_{\tau}}$ is the average $\tau$ neutrino energy.

Depending on the detector medium, we can normalize the total number of events by

$$
N_{\text {rock }}=\int C_{\text {rock }}(t) d t=\frac{\text { events }}{\text { effective detector mass }} .
$$

For different rocks consisting of $\mathrm{NaCl}, \mathrm{CaCO}_{3}$, and $\mathrm{SiO}_{2}$, we have calculated the expected neutron count rates for the SNBO type configuration for a supernova at $10 \mathrm{kpc}$. Figure 5 a shows results of these computations for total neutron production cross sections for three different rocks (Figure 3). These calculation assume zero neutrino mass for all species. These signal "history" curves show all the essential characteristics outlined as expected in an stellar collapse model: rapid rise to the maximum count rate within $150 \mathrm{msecs}$, and a long time scale decay after the peak. Clearly the event depends on the rock composition. 
Figure $5 \mathrm{~b}$ shows the effect of finite $\nu_{\mu}$ or $\nu_{\tau}$ masses on the expected signal count rate for a $\mathrm{NaCl}$ detector medium. These signal count rate curves show the characteristic massive neutrino effects such as flattening, smoothing, broadening and a slower time decrease in the signal. As Figure 5b indicates that a cosmologically significant $\tau$ neutrino mass may then be easily discernable. This is because for $m_{\nu_{\tau}}=10 \mathrm{eV}$ the rise time of the signal in the SNBO detector is delayed at least $\sim 0.1 \mathrm{sec}$ for a galactic supernova neutrino burst. As we mentioned in the introduction, the $\bar{\nu}_{e}$ signal from the Super Kamiokande detector should allow a good timing mark for the events.

Our analysis assumes that neutrino masses are less than the cosmological limit, $92 \mathrm{eV}$. If the $\nu_{\mu}$ or $\nu_{\tau}$ are heavier than a few hundred $\mathrm{eV}$, then their signal will be so spread out as to be difficult to interpret and pick out, due to detector background. For the low backgrounds indicated for the WIPP site, time of flight mass determination for $\nu_{\mu}$ or $\nu_{\tau}$ should be feasible for neutrino masses between about $90 \mathrm{eV}$ and $15 \mathrm{eV}$. The lower limit might conceivably be extendable down to of order $5 \mathrm{eV}$, depending on the reliability of our predicted supernova model.

Neutrino flavor mixing represents a complicating aspect for the time of flight decay measurement for $\nu_{\mu}$ and $\nu_{\tau}$ masses outlined above. In particular, if the massive high energy $\nu_{\mu}$ or $\nu_{\tau}$ undergoes a matter enhanced level crossing anywhere in the supernova above the neutrino sphere, then the detector may not be capable of resolving the expected time of flight decay. For example if $m_{\nu_{\tau}} \gtrsim 15 \mathrm{eV}$, and somewhere in the supernova $\nu_{\tau}$ is efficiently converted to $\nu_{e}$, then approximately one quarter of the neutron events induced by neutral current interactions will come from the resulting high energy $\nu_{e}$. Futhermore, in this case only one quarter of the neutrinos $\left(\bar{\nu}_{\tau}\right)$ responsible for the neutron events would propagate from the supernova to the detector in the high mass, time of flight delayed, eigenstate. This is to be contrasted with the case with no flavor mixing where fully one half the neutron events are caused by time of flight delayed neutrinos (e.g., both $\nu_{\tau}$ and $\bar{\nu}_{\tau}$ ). Estimates for SNBO show that mass determination is unlikely where only $1 / 4$ of the events are time delayed. 
Neutrino flavor interconversion depends not only on the neutrino energy and mass and the supernova density profile (these quantities determine the radius of a neutrino mass level crossing), but also on the vacuum mixing angle between $\nu_{\mu(\tau)}$ and $\nu_{e}$. Matter enhanced neutrino flavor oscillations in supernovae are discussed in reference [12] and in reference [29]. Reference [12] show that nucleosynthesis consideration mitigate against efficient $\nu_{\mu}$ or $\nu_{\tau} \rightarrow \nu_{e}$ flavor conversion for $\nu_{\mu}$ or $\nu_{\tau}$ with cosmologically significant masses. Reference [29] shows how efficient $\nu_{\mu(\tau)} \rightarrow \nu_{e}$ conversion for any $\nu_{\mu(r)}$ mass will have a readily discernable signature in a large water-detector like SK. This is signature is due to the ${ }^{18} \mathrm{O}\left(\nu_{e}, e^{-}\right){ }^{16} \mathrm{~F} \nu_{e}$ capture reaction, which has an effective $\nu_{e}$-energy-threshold of $35 \mathrm{MeV}$ or so, and produces a backward peaked electron [35]. Observation of this feature is a dead give a way that efficient $\nu_{\tau(\mu)} \leftrightarrow \nu_{e}$ interconversion has occurred in the supernova. Note that such an observation does not tell us what the neutrino mass scales are. For example, $\delta m^{2} \approx 10^{-6} \mathrm{eV}^{2}$ with the small mixing angle value for the preferred MSW solution of the solar neutrino problem would give efficient $\nu_{\tau(\mu)} \rightarrow \nu_{e}$ conversion in the envelope of the supernova [29], and should give a clear oxygen signal in SK.

Clearly our interpretation of the neutral current based detector data for a supernova burst event in the galaxy will depend on whether an oxygen $\nu$-conversion signal is seen in a concurrently operating water detector like SK. Table 3 shows the interpretation of possible outcomes for neutrino burst detection in SK and SNBO. For example if we both observe a time of flight delay signal in SNBO and an $\nu_{\tau(\mu)} \rightarrow \nu_{e}$ mixing signal in SK, then we know there is a massive $\nu_{\tau}$ or $\nu_{\mu}\left(m_{\nu_{\tau(\mu)}}>5 \mathrm{eV}\right)$ and that there is a matter enhanced level crossing. This could be consistent with MSW in the sun if the $\nu_{\tau}$ is the heavy neutrino and the matter enhanced level crossing in the supernova is $\nu_{r} \rightarrow \nu_{e}$ with $m_{\nu_{\mu}} \simeq 10^{-3} \mathrm{eV}$.

On the other hand if no time of flight delay is observed in SNBO, but a $\nu$-mixing signal is observed in SK, then it still might be that $\nu_{\mu}$ or $\nu_{\tau}$ has a mass $m_{\nu_{\tau(\mu)}}>5 \mathrm{eV}$, but the massive neutrino may have propagated to earth in the $\nu_{e}$ state due to efficient $\nu_{\tau(\mu)} \rightarrow \nu_{e}$ conversion. These signals could be consistent with the MSW solution in the sun. 
If a time of flight signal is seen in SNBO, but is not accompanied by a mixing signal in SK, then we conclude that there is a massive $\nu_{\mu}$ or $\nu_{\tau}$, but mixing between these species and $\nu_{e}$ is negligible. In particular, MSW is not the solution for the solar neutrino problem. The case where no time of flight signal is observed in SNBO and no mixing signal is observed in SK is similar: MSW can not be the solution of the solar neutrino problem; but we do place upper limits on the $\nu_{\mu}$ and $\nu_{\tau}$ masses which are far better than current experimental limits.

\section{Conclusions}

We have discussed the feasibility of detecting cosmologically significant neutrino masses using the time of flight delay technique for a neutral current based detector. Inelastic neutrinonuclei scattering and neutron detection provides an attractive technique for supernova neutrino detection. The inherent cut-off energy in such a detector will imply preferred sensitivity to $\nu_{\mu}$ and $\nu_{r}$. We have shown that such a detector operating in conjunction with a water Čerenkov detector such as SK can give valuable, and otherwise unobtainable, constraints on neutrino masses and mixings. We conclude that reliable limits on cosmologically significant $\nu_{\mu}$ and/or $\nu_{\tau}$ masses derived from galactic supernova burst events are feasible. Obtaining these limits depends on having neutral-current-based detectors operating in conjunction with the next galactic generation of water Čerenkov detectors. 


\section{References}

1. R. Cowsik and J. McCelland, Phys. Rev. Lett 29,669 (1972); J.E. Gunn, B.W. Lee, I. Lerche, D.N. Schramm, and G. Steigmann, Astrophys. J. 223, 1015 (1978); J.R. Bond, G.E. Efstathiou, and J. Silk, Phys. Rev. Lett 45, 61 (1980); G.R. Blumenthal, S.M. Faber, J.R. Primack, and M. Rees, Nature 311, 517 (1984).

2. G.F. Smoot et al. , Astrophys. J., 396, L1 (1992); E. Wright, et al. , Astrophys. J, 396, L13 (1992).

3. S.J. Maddox, et al. , Mon. Not. R. Astron. Soc., 242, 43 (1990).

4. R.K. Schaefer and Q. Shafi, Nature 359, 199 (1992); For a recent review on the Cold + Hot Dark Matter model scenario, see J.R. Primack's summary talk given at $I V$ Rencontres de Blois, on Particle Astrophysics, preprint SCIPP 92/51.

5. J.N. Bahcall and M.H. Pinsoneault, Rev. Mod. Phys., 64, 885 (1992).

6. see J.P. Revol, CERN-PPE/93-84, talk given at the Recontres de Physique de la Vallee d'Aoste 1993.

7. L. Wolfenstein, Phys. Rev. D17, 2369 (1978); S.P. Mikheyev and A. Yu. Smirnov, Yad. Fiz. 42, 1441 (1985).

8. M. Gell-Mann, P. Ramond and R. Slansky, in Supergravity ed. by Van Nieuwenhizen and D.Z. Freedman (North Holland, 1979)T. Yanagida, Proc. of the Workshop on Unified Theory and Baryon Number of the Universe, (Tsukuba, Ikabari, Japan, 1979).

9. H. Backe, et al., in Third Int. Sym. on Weak \& Electromagnetic Interactions in Nuclei, Dubna June 1992..

10. NOMAD collab., preprint CERN-SPSC/91-21 (1991).

11. CHORUS collab., preprint CERN-SPSC/90-42 (1990).

12. Y.Z. Quian, et al. , Phys. Rev. Lett. 71, 1965 (1993).

13. K. Hirata, et al. , Phys. Rev. Lett. 58,1490 (1987).

14. R.M. Bionta et al. , Phys. Rev. Lett. 58, 1494 (1987). 
15. D.B. Cline, G.M. Fuller, W.P. Hong, B.S. Meyer, and J. Wilson, UCLA-APH-1/90 (Unpublished).

16. A. Burrows, D. Klein, and R. Gandhi, Phys. Rev. D45,3361 (1992).

17. G.T. Ewan, in Proceeding of Supernova Watch Workshop, Santa Monica, California, 1990 (Unpublished).

18. As shown by A.Burrows et al. , in Ref.[13], the signal from neutral current events is not quite well discernable due to the total "apparent" deutron break up rate.

19. D. Seckel, G. Steigman, and T.P. Walker, Nucl. Phys. B336, 233 (1991).

20. R.G.H Robertson, T.J. Bowles, G.J. Stevenson, J.R., D.L. Wark, J.F. Wilkerson, and D.A. Knapp, Phys. ReV. Lett. 67, 957 (1991).

21. D.B. Cline, et al. , Proposal to study a new type of neutrino burst detector 1991 (Unpublished); For a recent progress, see a talk given at the International Conference on Neutrino Astrophysics, (Taka yama/Kamioka, Japan, Oct. 1992).

22. See for an example, M. Aglietta, et al. , Nuovo Cimento C12, 75 (1989).

23. D.B. Cline, invited given at the International Conference on Neutrino Astrophysics, Takayama/Kamioka, Japan, Oct. 1992, UCLA-APH0062-11/92.

24. J.N. Bahcall, M. Baldo-Ceolin, D. Cline and C. Rubbia, Phys. Lett. B178, 324 (1986); C. Rubbia, CERN-EP Internal Report 77-8 (1977); CERN-Harvard-Milano-Padova- RomaTokyo-Wisconsin Collaboration, INFN/AE-85/7, Frascati (1985); ICARUS Collaboration, ICARUS I: An Optimal Real Time Detector of Solar Neutrinos, LNF-89/005 (R)(1989).

25. T.W. Donnelly, Phys. Lett. B43, 93 (1973); J.D. Waleka., 1975 "Muon Physics", Vol. 2., (1975)ed. Hughes, V.W. and Wu. C.S. (Academic Press, New York); M. Fukugita, Y. Kohyama and Y. Kubodera, LAS, Preprint (1988); W.C. Haxton, Phys. Rev. C37, 2660 (1988); W.C. Haxton, Phys. Rev. Lett. 60, 1999 (1988).

26. G.M. Fuller and B.S. Meyer, Astrophys. J., 376, 701 (1991).

27. S.E. Woosley, P.A. Pinto and D. Hartman, Astrophys. J., 346, 395 (1989). 
28. D. Boyd at Ohio Sate University has recently measured the ambient neutron background in the underground WIPP site. His results indicate that the WIPP site would be a suitable place for the proposed SNBO detector.

29. Y.Z. Quian and G.M. Fuller, submitted to Phys. Rev. D.

30. J.R. Wilson, In Numerical Astrophysics, edited by J. Centrella, J. LeBlanc, and R. Bowers (Jones and Bartlett, Boston, 1985), P. 422.

31. R. Mayle and J. Wilson. UCRL-97355, Lawrence Livermore Laboratory and in Proceeding of the 5th Marcel Grossman Conference, World Scientific, eds. Blair, Buckingham, 217 (1989).

32. S. Bruenn, Phys. Rev. Lett. 59, 938 (1987).

33. E. Myra and A. Burrows, Astrophys. J. 364, 222 (1989).

34. A. Burrows, Astrophys. J. 334, 891 (1988).

35. W.C. Haxton, Phys. Rev. D 36, 2283 (1987). 


\section{Figure Captions}

Figure 1: Time evolution of luminosities for three neutrino flavors. Notice that the luminosities coincide with each other at very early times

Figure 2: $\nu+A(N, Z)$ inelastic scattering cross section averaged over a black body Fermi-Dirac distribution of neutrinos. We show results for ${ }^{28} \mathrm{Si}$ and ${ }^{23} \mathrm{Na}$ as indicated.

Figure 3: Neutron production cross sections for three different rocks. The cross sections for each rock are obtained by summing the individual nuclear cross sections weighted according to their frequency of occurrence. The $\bar{\nu}_{e}+p \rightarrow n+e^{+}$cross section is compared with neutron production cross sections. Above $E_{\nu_{\tau}} \sim 20 \mathrm{MeV}$, the $\mathrm{NaCl}, \mathrm{CaCO}_{3} \mathrm{SiO}_{2}$ cross sections are greater than the dominant water Čerenkov detector interaction.

Figure 4: Time evolution of average energies for three neutrino flavors are shown. In these calculations we fit the curves by polynomials to obtain smooth function.

Figure 5a: Signal count rates for three rocks.

Figure 5b: Effects of massive $\mu$ or $\tau$ neutrino $(7,10,20,40 \mathrm{eV})$ on the signal count rates for $\mathrm{NaCl}$ medium. 


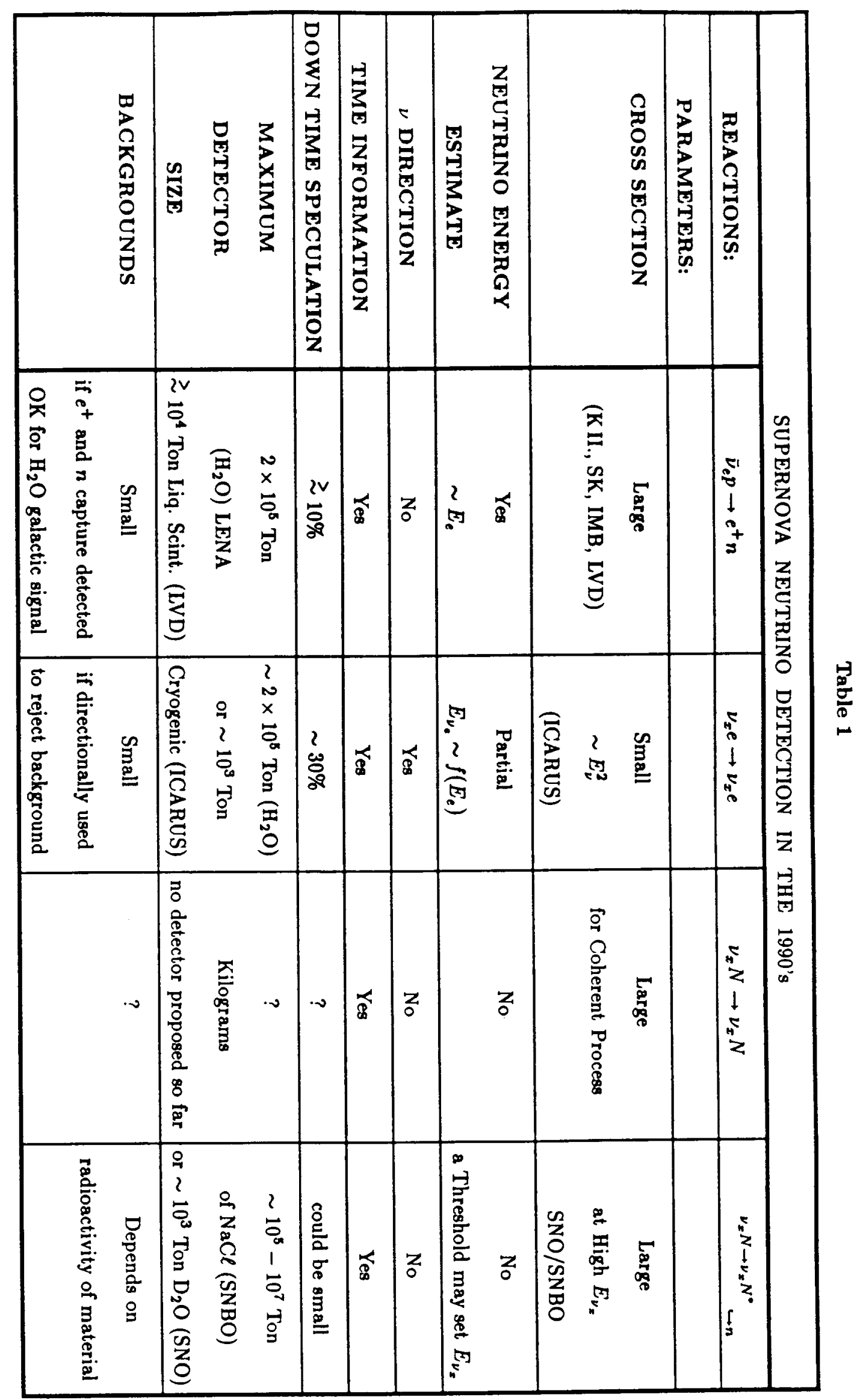




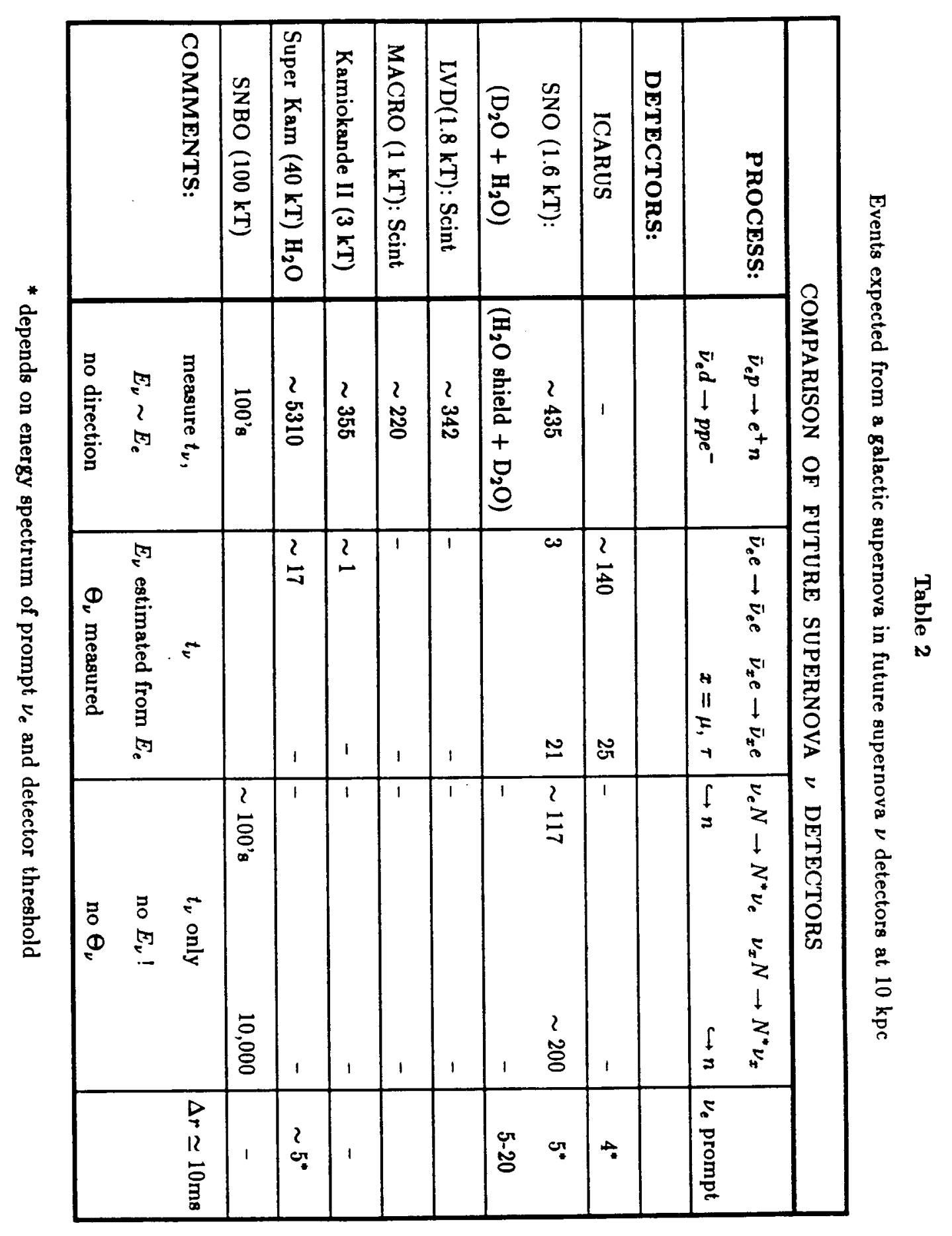




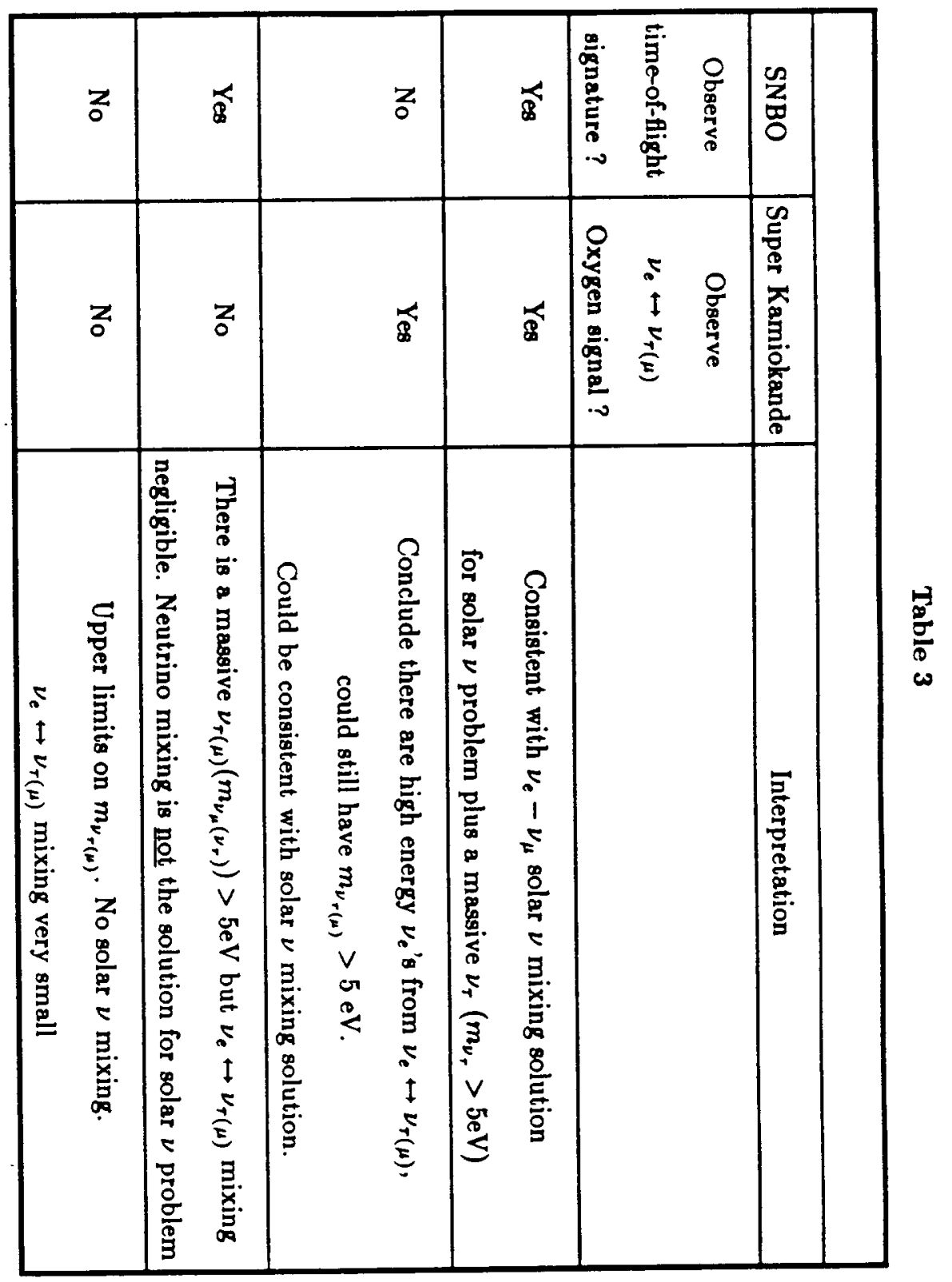


Intensity (10 $51 \mathrm{ergs} / \mathrm{sec})$

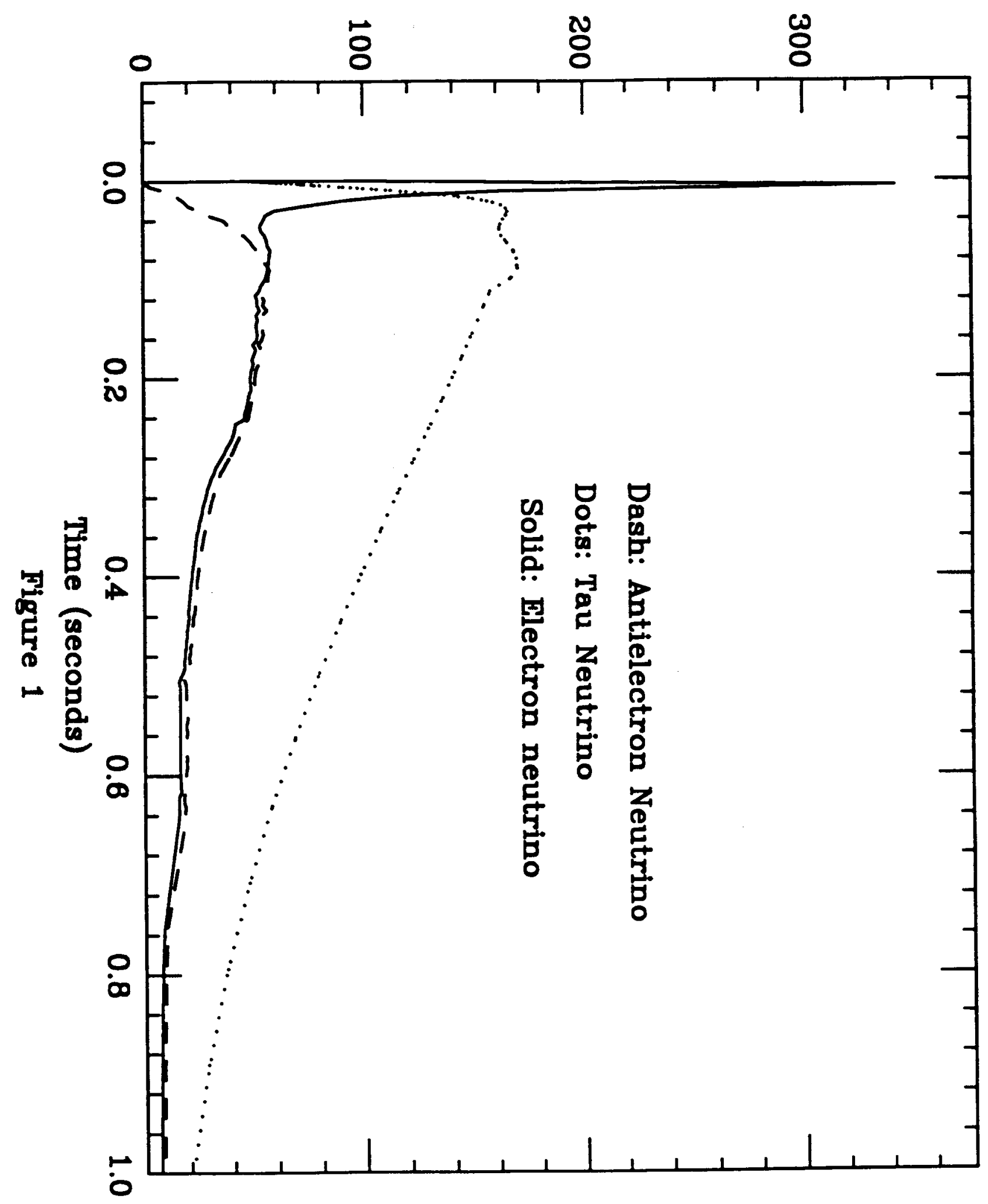




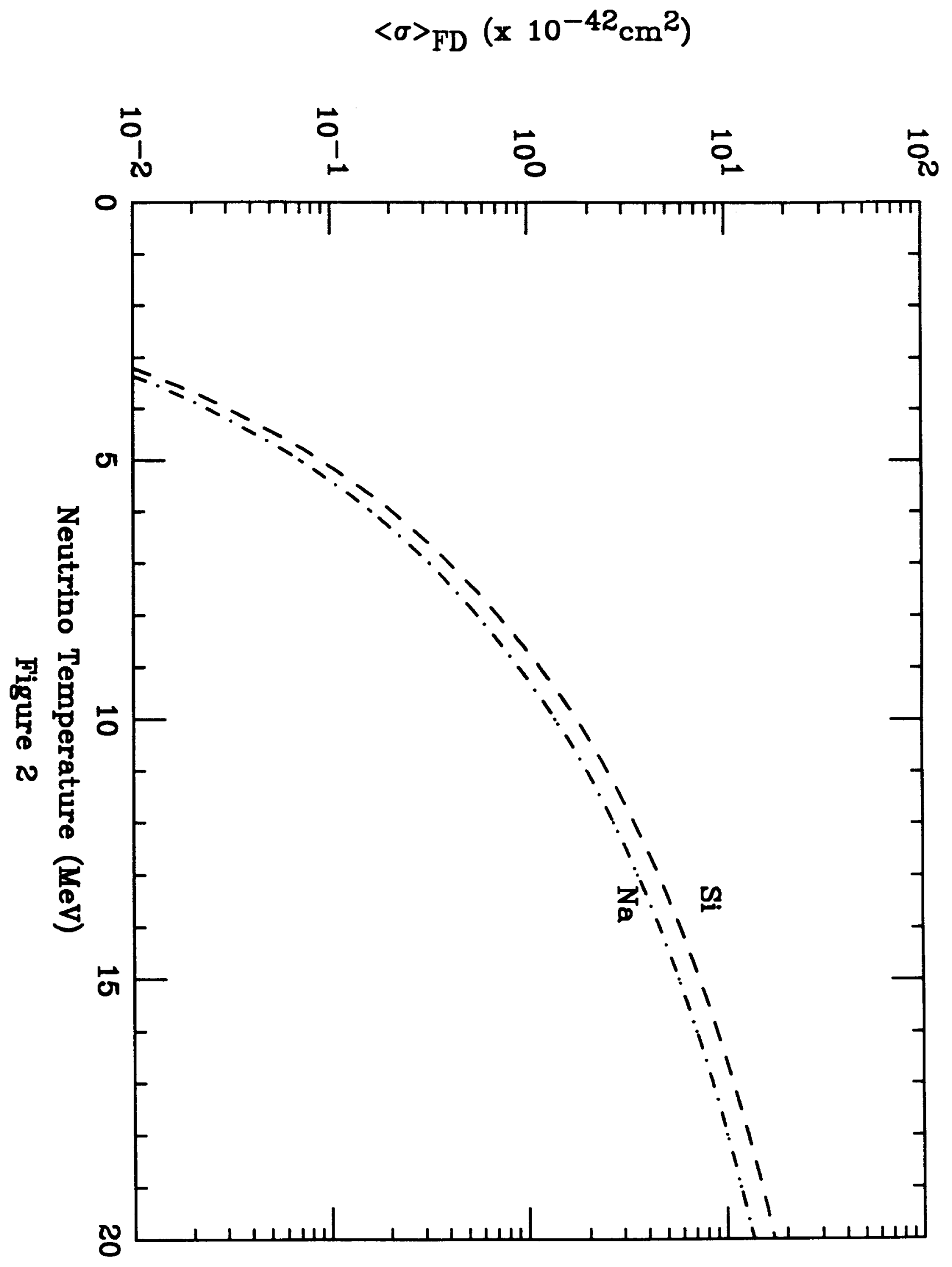


Cross Section (x $\left.10^{-42} \mathrm{~cm}^{2}\right)$

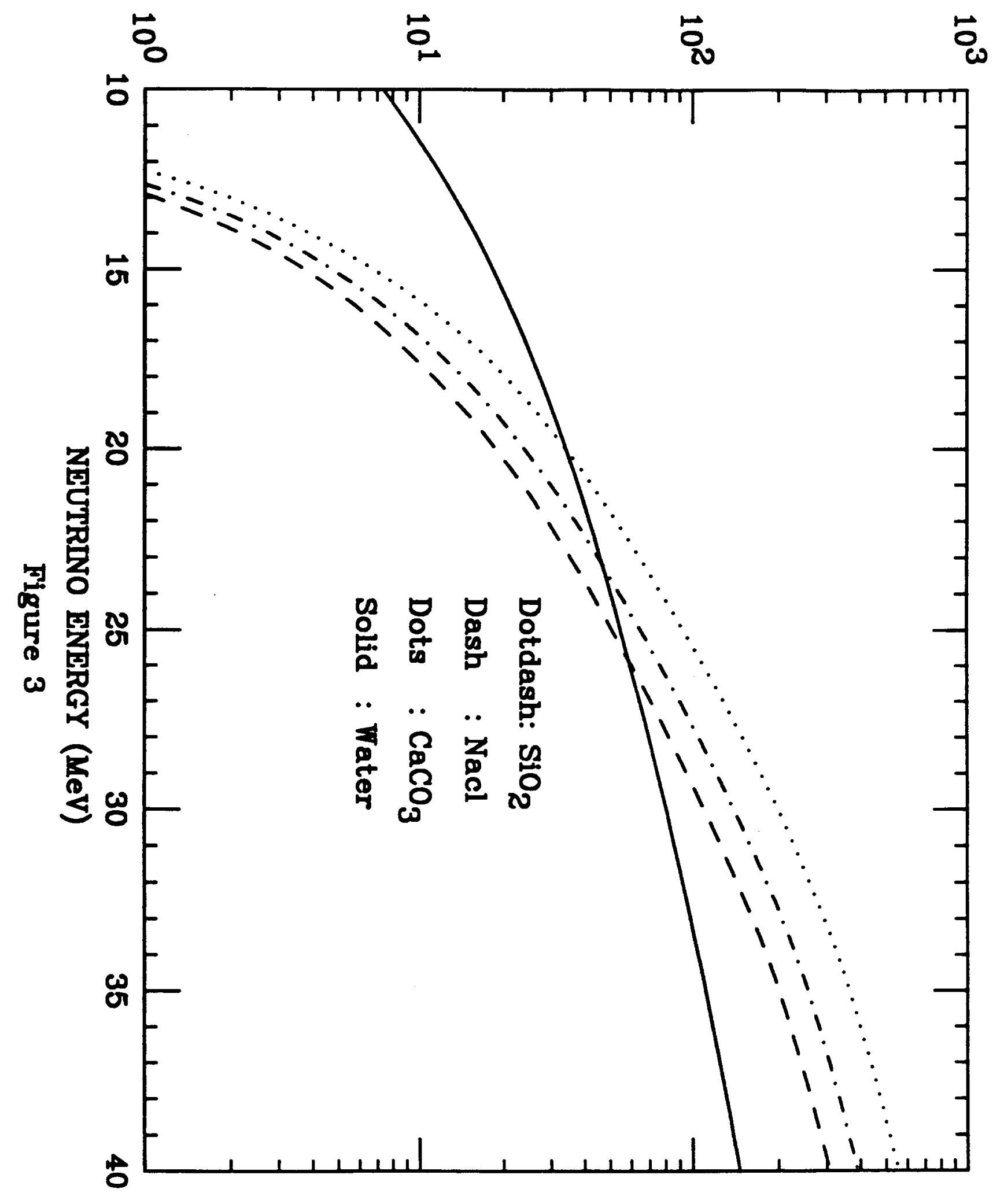


Average Neutrino Energy (MeV)

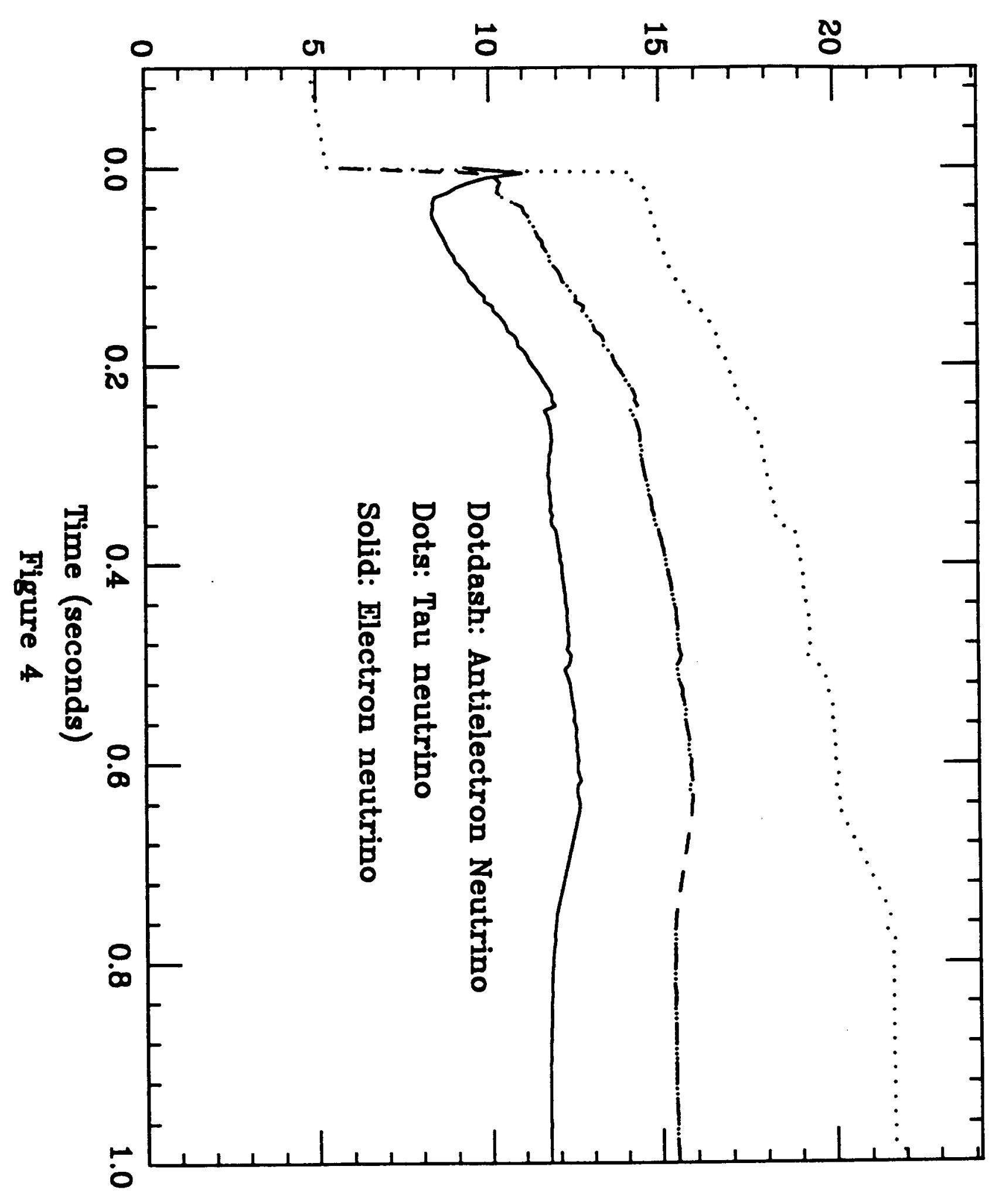


Count Rate (Hz)

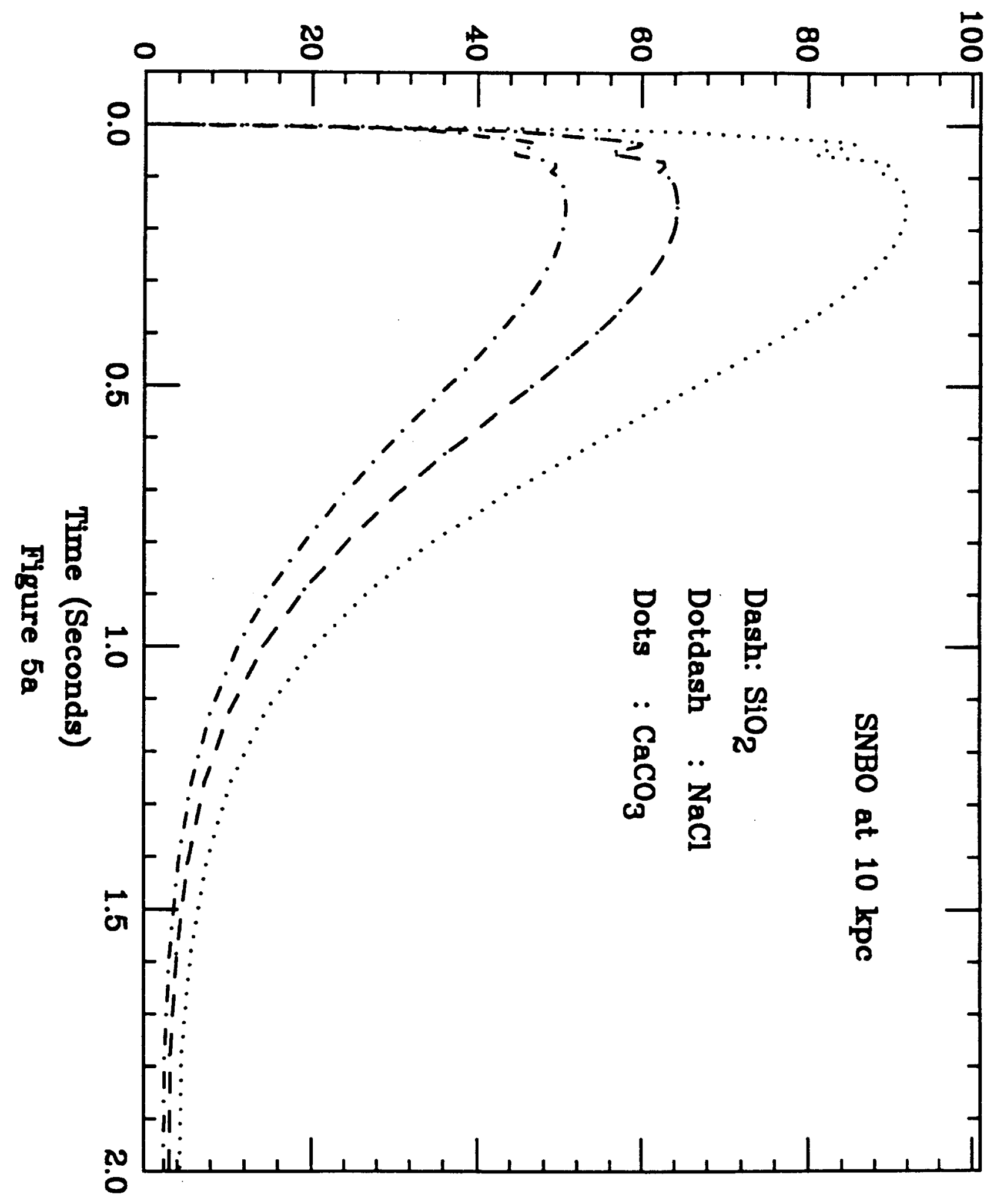


Neutron Count Rates (Hz)

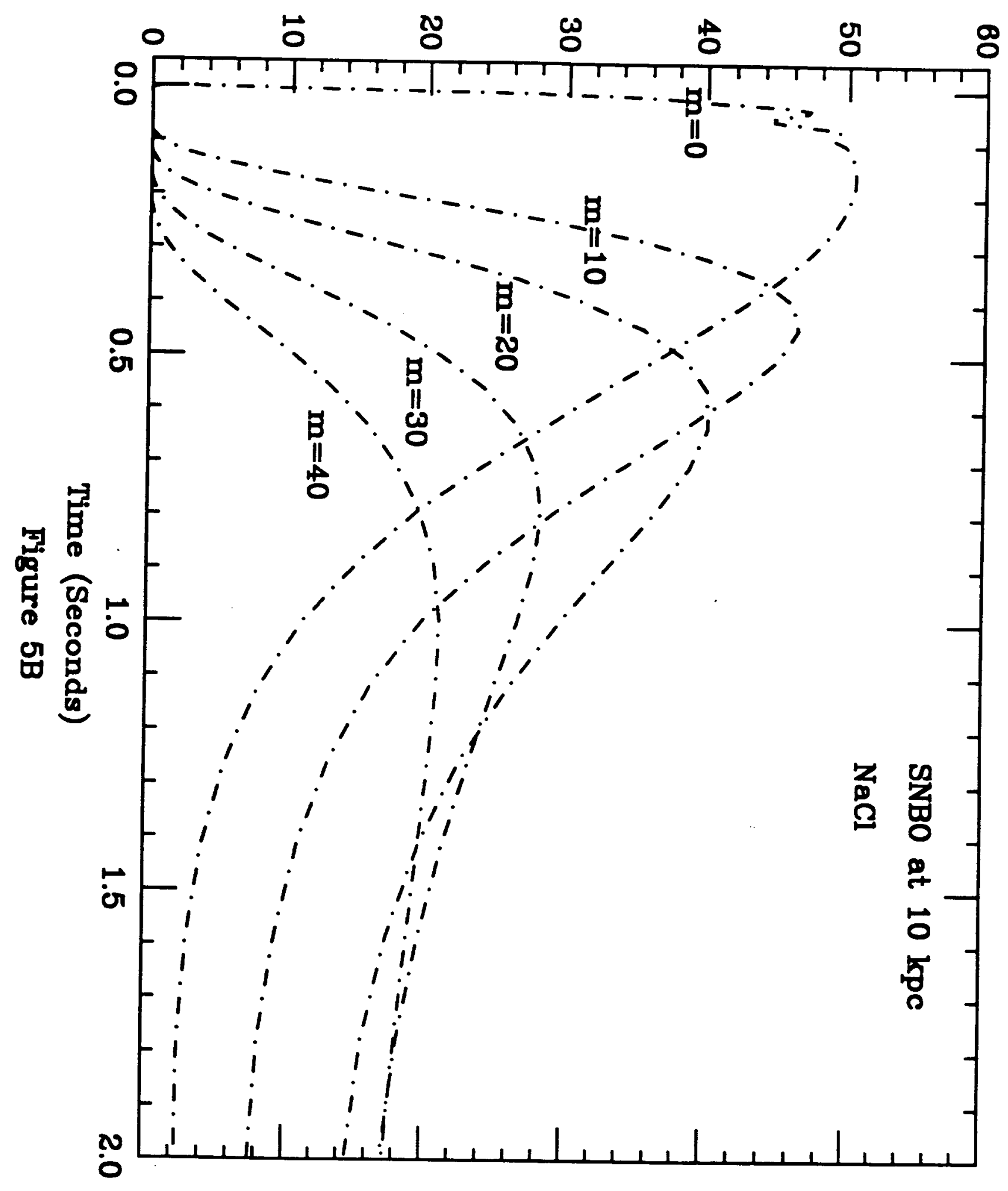

